\title{
Effects of tendon vibration and age on force reproduction task performed with wrist flexors
}

\author{
Mélanie Henry ${ }^{1} \cdot$ Alp Eşrefoğlu $^{1} \cdot$ Jacques Duchateau ${ }^{1} \cdot$ Stéphane Baudry ${ }^{1}$ (I)
}

Received: 23 October 2021 / Accepted: 17 January 2022

(c) The Author(s), under exclusive licence to Springer-Verlag GmbH Germany, part of Springer Nature 2022

\begin{abstract}
The sense of force is suggested to rely in part on proprioceptive inputs when assessed with a force reproduction task. The age-related alterations in proprioceptive system could, therefore, alter the sense of force. This study investigated the effects of tendon vibration on a force reproduction task performed with the wrist flexors in 18 young (20-40 year) and 18 older adults (60-90 year). Participants matched a target force (5\% or $20 \%$ of their maximal force) with visual feedback of the force produced (target phase), and reproduced the target force without visual feedback (reproduction phase) after a 5-s rest period with or without vibration. The force reproduction error was expressed as the ratio between the force produced during the reproduction and the target phases. For the trials with vibration, the error was expressed as the ratio between the force produced during the reproduction phase performed with and without vibration. Tactile acuity was assessed with a two-point discrimination test. The error was greater at $5 \%$ than at $20 \%$ contraction intensity $(p<0.001)$, and in older $[56.5(32.2) \%$; mean (SD)] than in young adults [33.5 (13.6)\%] at $5 \%(p=0.002)$ but not $20 \%$ target $(p=0.46)$. Tendon vibration had a greater effect at $5 \%$ than $20 \%$ contraction intensity, and in older [41.7 (32.4)\%, $p<0.001$ ] than young adults [20.0 $(16.1) \%$ ]. Tactile acuity was lesser in older than young adults $(p<0.001)$. The results support the contribution of proprioception in the sense of force, and highlight a decrease in performance with ageing restricted to low-force contractions.
\end{abstract}

Keywords Sense of force $\cdot$ Proprioception $\cdot$ Muscle afferents $\cdot$ Electromyography

\section{Introduction}

Successful performance of motor tasks requires the adequate modulation of force output, which depends, in part, on the integration of information from various mechanoreceptors located in muscles, muscle-tendon interfaces, and joints. These signals form the basis for the perception of self-movement and body position, which is termed proprioception and defined as the conscious or unconscious perception of the position of different parts of the body in space. Along with the kinaesthetic sense (the senses of position and movement), the sense of force is another aspect

Communicated by Francesco Lacquaniti, MD.

Stéphane Baudry

stephanebaudry@ulb.be

1 Laboratory of Applied Biology, Research Unit in Applied Neurophysiology (LABNeuro), ULB-Neurosciences Institute (UNI), Faculty for Motor Sciences, Université Libre de Bruxelles (ULB), 808, Route de Lennik, CP 640, 1070 Brussels, Belgium of proprioception (Proske and Gandevia 2012). The sense of force represents the ability to correctly perceive and reproduce a given level of force. However, it is still debated whether the sense of force really relies on proprioceptive inputs. This may depend on the experimental approach used for its assessment. Indeed, it can be evaluated by asking the participants to match with one limb the force produced by the contralateral limb (two-arm matching task), or by asking to reproduce a force with the same limb (force reproduction task) (Proske and Allen 2019). It has been suggested that the two-arm matching task does not require proprioceptive feedback but rather a centrally generated signal (efference copy). In contrast, the force reproduction task is suggested to mainly rely on proprioceptive inputs, including signals from muscle spindles and Golgi tendon organs (Monjo et al. 2018; Proske and Allen 2019), in addition to sensory inputs from skin mechanoreceptors (De Serres and Fang 2004; Proske and Allen 2019).

Mechanical vibration applied to a distal tendon (local vibration; LV) could elucidate the implication of the proprioceptive signals from muscle sensors in the force 
reproduction task. When applied during a voluntary contraction, LV of sufficient amplitude $(0.5-1 \mathrm{~mm})$ and frequency $(>20 \mathrm{~Hz})$ can stimulate muscle spindles and Golgi tendon organs, inducing activity of Ia and II afferent fibres (muscle spindle afferents), and Ib afferent fibres (tendon organ afferents) (Burke et al. 1976; Roll et al. 1989; Fallon and Macefield 2007). The effect of LV on the proprioceptive senses has been documented for a long time (Goodwin et al. 1972), with alterations of the sense of position and movement, for example (Cordo et al. 1995). Recently, Boucher and colleagues (Boucher et al. 2015) investigated the effect of LV on a force reproduction task performed with the plantar flexor muscles. The task consisted of producing a submaximal isometric force, corresponding to $60 \%$ of their maximal force, as quickly as possible. After a period of learning, the participants were instructed to repeat the task while LV was applied. The poorer force reproduction performance when LV was applied was interpreted as a consequence of distortion of the proprioceptive signal. However, due to the nature of the task performed (ballistic contraction), which limits potential corrections of the force during the execution of the task, the results of Boucher et al. (2015) could only provide limited information on the effect of LV on the sense of force.

A relevant and well-known aspect of motor control is its decline with ageing, which appears to reflect dysfunctions of the sensory and motor components of the neuromuscular system (Hunter et al. 2016), including a decline in proprioceptive acuity (Henry and Baudry 2019). Motor performance deficits include alterations in maximal force (Klass et al. 2005), rate of force development (Klass et al. 2008) and force steadiness (Enoka et al. 2003). These deficits have a negative impact on the ability of older adults to perform functional activities of daily living. Two studies have investigated the effect of ageing on the force reproduction performance (De Serres and Fang 2004; Franco et al. 2015). In a pinch grip task performed with the index and the thumb, De Serres and Fang (2004) observed greater force reproduction errors in older than young adults at low $(5 \%$ of the peak force developed during a maximal voluntary contraction; MVC) but not moderate forces (20 and 40\% MVC). These results suggest an age-related difference in force reproduction performance, which could depend on the contraction intensity. Franco et al. (2015), however, did not report an age-related effect in a force reproduction task requiring to produce ankle dorsiflexion or plantar flexion force at two contraction intensities (10\% and 20\% MVC). These divergent results can reflect, in part, the different muscle groups investigated, with cortical motor and sensory areas contributing differently to isolated upper and lower limb movement (Luft et al. 2002). In addition, De Serres and Fang (2004) used a relative index of force error, with the error expressed as a percentage of the target force whereas Franco et al. (2015) reported the absolute difference between the target force and the force produced. Although these authors did not report the maximal force of their participants, it is well documented that ageing is accompanied by a decrease in maximal force capacity (Klass et al. 2005). Consequently, a similar absolute force error in young and older adults should not represent the same relative error. From these two studies, it is therefore not possible to draw a definitive conclusion on the effect of ageing on the sense of force.

The objectives of this study were to document the effects of LV and age on a force reproduction task performed with the wrist flexor muscles at two contraction intensities. The choice of this muscle group was based on previous work indicating alterations in force control in forearm muscles with ageing, especially for low contraction forces, with different neural adjustments between young and older adults (Baudry et al. 2010). Based on the assumption of a contribution of proprioceptive signals on force reproduction performance, we expected a poorer performance with LV. The decline in the integration of the proprioceptive signal with ageing (Goble et al. 2011, 2009; Mildren et al. 2020) should alter the performance in the force reproduction task in older adults, and make more challenging the discrimination and processing of the genuine proprioceptive signals originating from the muscle contraction from those arising from LV. Accordingly, we expected that LV should have a larger effect in older than young adults.

\section{Methods}

\section{Participants}

Eighteen young adults aged from 20 to 40 years [mean (SD); 28 (6) year; 9 females and 9 males] and 18 older adults aged from 60 to 90 years [71 (7) year; 11 females and 7 males], all physically active, volunteered to participate to the study after written informed consent was obtained. Approval for the project was obtained from the local Ethics Committee, and all procedures used in this study conformed to the Declaration of Helsinki.

Individuals with Parkinson's disease, multiple sclerosis, diabetes, stroke, cardiac history or neurological damage, taking medications that could influence motor control [sedatives, hypnotics, antidepressants and benzodiazepines (Woolcott et al. 2009)] or suffering from orthopaedic problems could not participate to this study. All participants but four ( 3 young and 1 older adult) were right-handed, as assessed by the Edinburgh Handed Inventory (Oldfield 1971). Participants were not allowed to consume caffeine during the last $12 \mathrm{~h}$ preceding the experiment, and were asked to refrain from doing intense physical activity for $24 \mathrm{~h}$ before testing. 


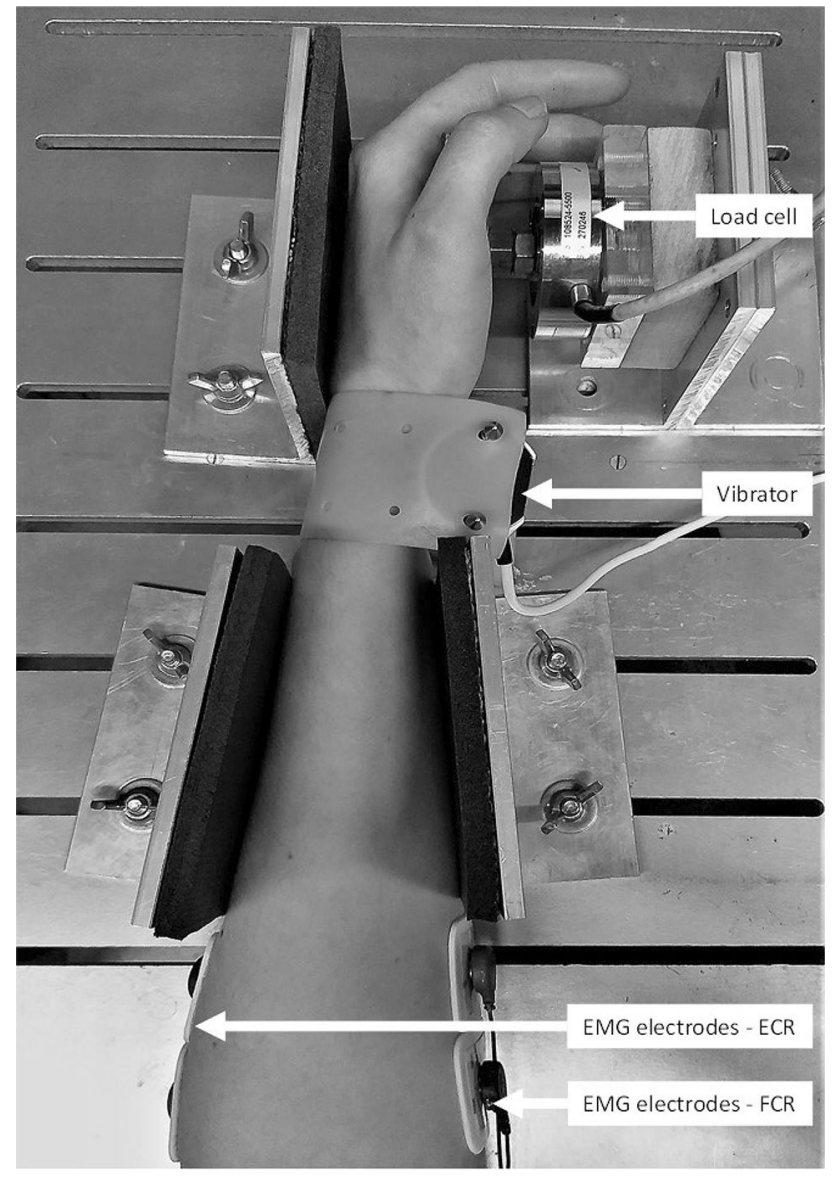

Fig. 1 Picture of the experimental set-up and placement of the EMG electrodes and the vibrator. $F C R$ flexor carpi radialis, ECR extensor carpi radialis

\section{Experimental setup}

Participants were seated in a chair with the forearm of the dominant arm placed on a custom-made ergometer. The wrist was in neutral position $\left(0^{\circ}\right.$ of flexion, midway between pronation and supination), while the shoulder and elbow were flexed at about $25^{\circ}$ and $40-50^{\circ}$ (elbow full extension: $\left.0^{\circ}\right)$, respectively. The palm of the hand was fixed against a pad ( $8 \mathrm{~cm}$ high and $3 \mathrm{~cm}$ wide) at the metacarpophalangeal joints. The pad was connected to a force transducer to record the force of the wrist flexors (Fig. 1). A screen was placed in front of the participants to provide them with visual feedback of the wrist flexion force produced.

\section{Surface electromyogram}

The surface electromyogram (EMG) was recorded from the flexor carpi radialis (FCR) and the extensor carpi radialis (ECR) with auto-adhesive electrodes (3 M, Red Dot ${ }^{\mathrm{TM}}$ ) placed in bipolar configuration with an inter-electrode (centre to centre) distance of $2 \mathrm{~cm}$. The location of the electrodes was determined by palpation and ultrasound imaging (DP-6900Vet; Shenzhen Mindray Bio-Medical Electronics), using a 6-cm width linear-array probe (7.5-MHz; 75L60EA, Shenzhen Mindray Bio-Medical Electronics), during wrist flexion, extension and abduction. The reference electrodes for FCR and ECR were placed over the olecranon and the humeral epicondyle, respectively. Before attaching the electrodes, the skin was shaved when necessary and cleaned with a solution of alcohol, ether, and acetone to reduce the impedance at the skin-electrode interface. EMG signals were amplified $(1000 \times)$ and band-pass filtered $(10-1000 \mathrm{~Hz})$ prior to A/D sampling at $2 \mathrm{kHz}$ (Power 1401, 16-bit resolution, Cambridge Electronic Design, UK) before being stored on a computer.

\section{MVC}

The MVC of the wrist flexors consisted of a gradual increase in force from zero to maximum within $2 \mathrm{~s}$ and sustained thereafter for 4-5 s. Three trials were performed, with participants resting for 90-120 s between trials to avoid fatigue. If the peak force of at least two of the three MVCs was within $5 \%$ of each other, the greatest value of these two MVCs was taken as the maximum. Otherwise, additional trials were performed until the 5\% criterion was achieved. Participants never had to perform more than five MVCs per session to reach this criterion. The greatest MVC force was used thereafter to adjust the target displayed on the monitor during the force reproduction task. The average value of the rectified EMG (aEMG) measured within a 1-s time window around the peak force was used for FCR EMG normalisation purpose. A single MVC was performed with the wrist extensors for ECR as the ergometer did not allow to record force in the wrist extension direction. The highest aEMG within 1 -s window was used for normalisation purpose.

\section{Local vibration}

Local vibration (LV) was applied using a mechanical vibrator (VB 115, Techno Concept, Mane, France) placed transversally to the distal tendons of the wrist flexor muscles, $\sim 2 \mathrm{~cm}$ proximal to the radial styloid process. The vibrator was held in place by means of a rubber band fastened around the wrist. The vibration was applied with an $80-\mathrm{Hz}$ frequency and a 1-mm amplitude.

\section{Two-point discrimination test}

As sensory inputs from mechanoreceptors of the skin could be involved in the force reproduction task (De Serres and Fang 2004; Proske and Allen 2019), tactile sensitivity was assessed by means of a two-point discrimination test over 

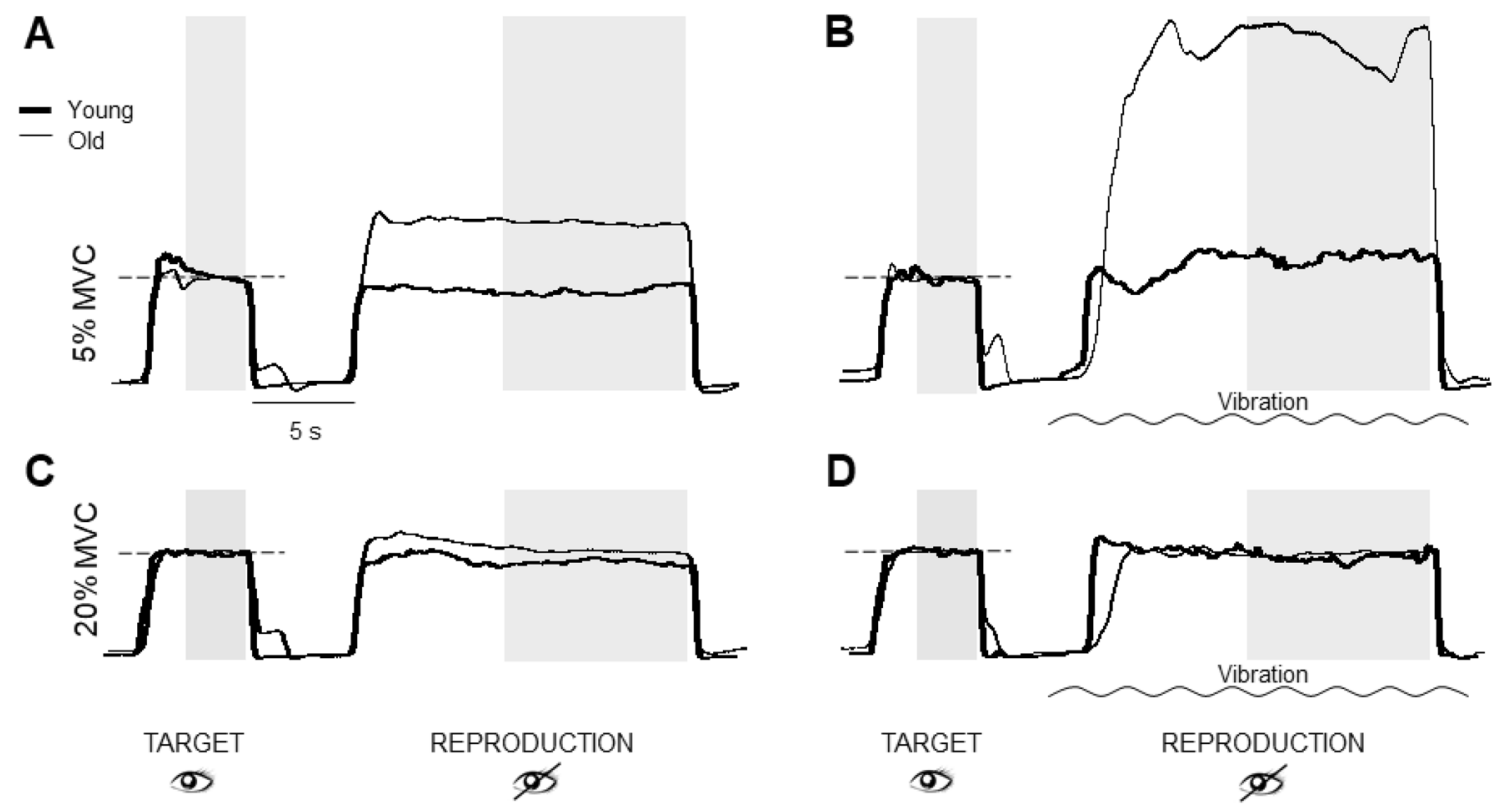

Fig. 2 Original traces of the force produced by one young (thick line) and one older (thin line) participant during a single force reproduction trial with $(\mathbf{B}, \mathbf{D})$ and without $(\mathbf{A}, \mathbf{C})$ local vibration performed at $5 \%(\mathbf{A}, \mathbf{B})$ and $20 \%(\mathbf{C}, \mathbf{D})$ of the maximal force produced during a maximal voluntary contraction (MVC). The thin horizontal dashed lines represent the target force. The undulatory lines represent the

the pulp of the index. This was done because fingertip assessment is more common, especially in medical routine, increasing the clinical relevance of this measurement. The two points of the tool were applied in a progressive order over the pulp of the index, parallel to the distal phalanx, for $1 \mathrm{~s}$. The participants, with their eyes closed, had to respond verbally about whether they felt one or two points. The test was performed three times, and the mean distance for which participants discriminated the two points of the tool was used as the tactile sensitivity score.

\section{Force reproduction task}

The force reproduction task consisted of two phases: the target (target) and the reproduction (reproduction) phases. During the target phase, participants had to match for $5 \mathrm{~s}$ the target forcedisplayed on the screen as a green horizontal line-with a red horizontal cursor representing the force they produced. The cursor moved vertically, with upward displacement corresponding to an increase in the force produced. During the target phase, participants were asked to focus their attention on the sensation of the force developed by the wrist flexors. The reproduction phase consisted of reproducing the target force during $15 \mathrm{~s}$, without visual feedback of the force nor the green horizontal line illustrating the target force. Target and reproduction phases were separated by a 5-s epoch during which the participants were asked to relax completely the

forearm muscles (Fig. 2). The force reproduction task was performed at two contraction intensities: $5 \%$ and $20 \%$ for the MVC force. Three trials were performed at each contraction intensity, with the order of the contraction intensities being counterbalanced across participants. For each contraction intensity, three additional trials were performed while applying LV during the reproduction phase. LV started prior to the onset of the reproduction phase and was applied throughout this phase performed without visual feedback of the force produced. Participants performed all the trials without LV prior to those with LV. Two successive force reproduction tasks were separated by at least $60 \mathrm{~s}$. No information was provided to the participants regarding their overall performance in the reproduction phase. Before starting the force reproduction task at one contraction intensity, three familiarisation contractions of $15 \mathrm{~s}$ with visual feedback of the force were performed to train the participants to focus on the sensation of the force produced by the wrist flexors while matching the target force.

To ensure that the amount of visual feedback provided was the same across subjects, the visual angle was set at $0.1^{\circ}$ by means of the following formula:

$a=2 * \tan ^{-1}$ (force fluctuations $/ d$ ),

where $a=$ visual angle, force fluctuations $=$ half of the amplitude of force fluctuations estimated at $3 \%$ of targeted force (Fox et al. 2013), and $d=$ distance of the eye to the computer 
screen. The choice of a small visual angle $\left(0.1^{\circ}\right)$ that did not magnify the force fluctuations helped the participants to focus on the sensation of force while matching the TARGET force.

\section{Data reduction}

The force reproduction performance was assessed by computing the absolute error (AE) and the signed error (SE) (De Serres and Fang 2004). AE provides information about the overall error, regardless of its direction, reflecting thereby the magnitude of the error. AE was calculated with the following formula, using the absolute value of the difference between the reproduction force and the target force: reproduction phase was also expressed as a percentage of the aEMG recorded during the target phase. The index of antagonist coactivation was calculated as the ratio between the aEMG of the antagonist (ECR) to the aEMG of the agonist (FCR), and the index of coactivation during the reproduction phase was also expressed as a percentage of the index calculated during the target phase. Because LV altered the EMG signal, EMG parameters were not analysed for the force reproduction task performed with $\mathrm{LV}$.

\section{Statistical analysis}

The JASP software (JASP version 0.13.1, Amsterdam, Netherlands) was used for statistical analysis. The

$\mathrm{AE}(\%)=1 / n \times \sum[\mid$ reproduction force - target force $/ /$ target force $\times 100]$,

where reproduction force is the mean force produced during the last $9 \mathrm{~s}$ of this phase, target force is the mean force produced during the last $3 \mathrm{~s}$ of the target phase, and $\mathrm{n}$ is the number of trials $(n=3)$. The last $9 \mathrm{~s}$ of the reproduction phase corresponded to the portion of the phase during which the force was stabilised around the force participants felt as the target one.

SE provides information about the direction of the error: a negative value indicated that participants undershot the target whereas a positive value indicated that participants overshot the target. SE was calculated with the same formula as $\mathrm{AE}$, using the real (signed) value of the difference between the reproduction force and the target force.

To assess the effect of LV on the force reproduction performance, the absolute error $\left(\mathrm{AE}_{\mathrm{LV}}\right)$ was calculated with the following formula:
D'Agostino and Pearson normality test confirmed that the distribution of each data set fitted a Gaussian function. Two-way ANOVAs (age $\times$ contraction intensity) were used to assess the effect of age and contraction intensity on $\mathrm{AE}$, $\mathrm{SE}, \mathrm{AE}_{\mathrm{LV}}, \mathrm{SE}_{\mathrm{LV}}$ and aEMG during the reproduction phase. When appropriate, Holm post-hoc tests were used to identify differences between means. In addition, aEMG during the TARGET phase at each contraction intensity, MVC force and tactile acuity were compared between young and older adults with Student's $t$ tests for independent samples. The level of statistical significance was set at $p<0.05$ for all comparisons. For each dependent variable, the results of ANOVAs are provided in Table 1. Values are expressed as mean (SD) in the text and tables and mean (SEM) in Fig. 3.

$A E_{L V}(\%)=1 / n \times \sum\left[\mid\right.$ reproduction $_{\mathrm{LV}}$ force - reproduction force $\mid /$ reproduction force $\left.\times 100\right]$,

where reproduction $_{\mathrm{LV}}$ force is the mean force produced in the last $9 \mathrm{~s}$ during the reproduction phase with $\mathrm{LV}$, reproduction force is the averaged value of the force produced for the three trials without LV during the last $9 \mathrm{~s}$ of the phase, and $\mathrm{n}$ is the number of trials $(n=3)$. The signed error during $\mathrm{LV}$ trials $\left(\mathrm{SE}_{\mathrm{LV}}\right)$ was calculated with the same formula as $\mathrm{AE}_{\mathrm{LV}}$ except that the real (signed) value of the difference between reproduction ${ }_{\mathrm{LV}}$ and reproduction forces was used. Accordingly, positive values indicated a worsening of the reproduction performance relative to the trials without LV.

The aEMG of FCR and ECR was measured over the same epoch as for the force signal and normalised to aEMG value measured during the MVC. The aEMG during the

\section{Results}

The MVC force was greater in young [189.1 (85.1) $N]$ compared with older adults [121.0 (30.4) $N ; p=0.003]$. The score of the two-point discrimination test was greater in older [3.4 (1.0) $\mathrm{mm}]$ than in young adults $[2.4(0.4) \mathrm{mm}$, $p<0.001]$, indicating a lower tactile acuity in older adults.

\section{Force reproduction task}

The AE was greater for the 5\% than for the $20 \%$ contraction intensity $(p<0.001)$, and was greater in older than in young adults $(p=0.007)$. Furthermore, age $\times$ intensity interaction $(p=0.031)$ and associated post-hoc tests indicated that $\mathrm{AE}$ was greater in older than young adults for the $5 \%(p=0.002)$ 
Table 1 Mean (SD) of the main variables with the results of the analyses of variance (ANOVA)

\begin{tabular}{|c|c|c|c|c|c|c|c|}
\hline \multirow[t]{2}{*}{ Variables } & \multicolumn{2}{|l|}{ Young } & \multicolumn{2}{|l|}{ Older } & \multicolumn{3}{|c|}{ Analysis of variance (ANOVA) } \\
\hline & $5 \% \mathrm{MVC}$ & $20 \% \mathrm{MVC}$ & $5 \% \mathrm{MVC}$ & $20 \% \mathrm{MVC}$ & Age & Intensity & Age $\times$ intensity \\
\hline $\mathrm{AE}(\%)$ & $33.5(13.6)$ & $13.6(8.7)$ & $56.5(32.2)$ & $18.2(11.0)$ & $F=8.4, p=0.007^{*}$ & $F=50.9, p<0.001^{*}$ & $F=5.1, p=0.031 *$ \\
\hline SE $(\%)$ & $31.9(14.9)$ & $5.9(13.4)$ & $46.4(45.9)$ & $-1.4(21.1)$ & $F=0.2, p=0.65$ & $F=61.5, p<0.001^{*}$ & $F=5.3, p=0.027^{*}$ \\
\hline $\mathrm{AE}_{\mathrm{LV}}(\%)$ & $27.6(19.3)$ & $12.4(5.9)$ & $58.7(35.0)$ & $24.8(18.1)$ & $F=15.5, p<0.001 *$ & $F=25.1, p<0.001 *$ & $F=3.6, p=0.066$ \\
\hline $\mathrm{SE}_{\mathrm{LV}}(\%)$ & $17.6(28.2)$ & $-4.9(10.6)$ & $52.2(43.7)$ & $20.6(22.2)$ & $F=16.9, p<0.001^{*}$ & $F=19.1, p<0.001 *$ & $F=0.5, p=0.47$ \\
\hline FCR (\% target) & $32.4(41.1)$ & $14.7(40.2)$ & $32.4(41.7)$ & $6.2(31.2)$ & $F=0.8, p=0.38$ & $F=23.4, p<0.001^{*}$ & $F=0.4, p=0.54$ \\
\hline ECR ( $\%$ target $)$ & $5.7(7.2)$ & $29.7(28.1)$ & $8.2(13.5)$ & $13.9(32.2)$ & $F=1.7, p=0.20$ & $F=6.7, p=0.014 *$ & $F=2.5, p=0.12$ \\
\hline Coactivation (\% target) & $-3.6(18.8)$ & $15.4(26.8)$ & $-5.6(13.8)$ & $14.5(29.6)$ & $F=0.1, p=0.79$ & $F=12.1, p=0.001^{*}$ & $F=0.0, p=0.92$ \\
\hline
\end{tabular}

Values are expressed as mean (SD)

$S E$ and $A E$ signed and absolute force reproduction error, respectively, $S E_{L V}$ and $A E_{L V}$ signed and absolute force reproduction error with local vibration, respectively, FCR and ECR aEMG of flexor carpi radialis and extensor carpi radialis, respectively, during the reproduction phase (expressed as a percentage of the target phase)

*Denotes statistically significant main effects or interactions $(p<0.05)$

Fig. 3 Individuals absolute error (AE) and signed error (SE) during the reproduction tasks performed without local vibration (A and $\mathbf{C}$, respectively) and with local vibration (LV; B and D) for young (open circles) and older adults (filled circles). The error during LV trials are expressed relative to the error measured during trials without LV. Accordingly, positive values for trials with LV indicate a poorer performance relative to the trials without LV. Each point represents the mean value of the three trials performed within each experimental condition. ** and $* * *$ denote statistical differences at $p<0.01$ and $p<0.001$, respectively (see the text for more details)
A

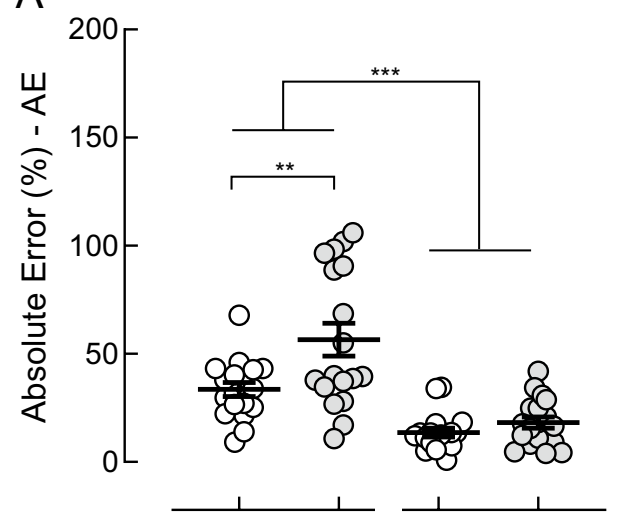

C

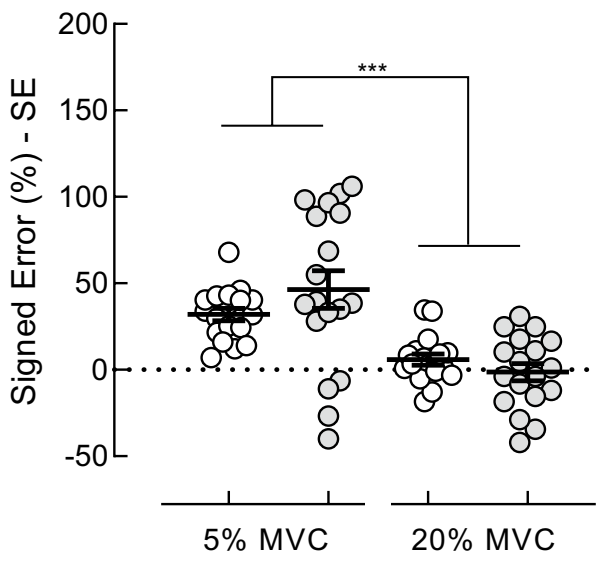

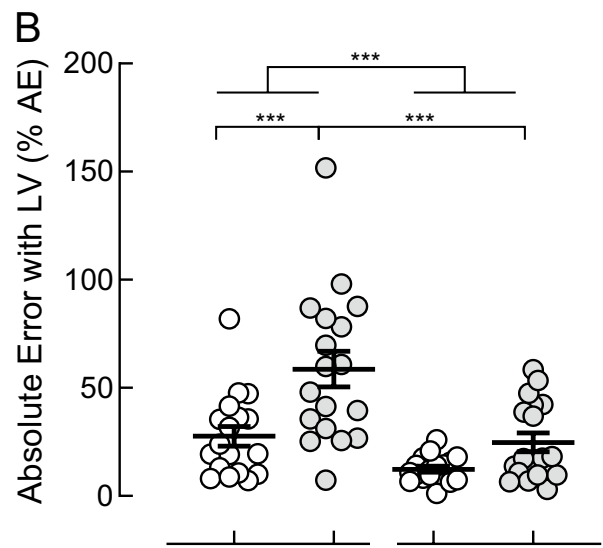

D
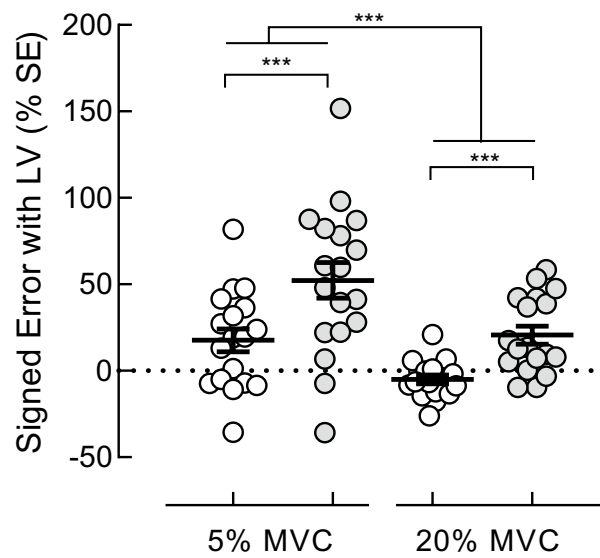

Young o O Older

but not the $20 \%$ contraction intensity ( $p=0.46$ ) (Fig. $3 \mathrm{~A}-\mathrm{C}$ ). The SE was greater for the $5 \%$ than the $20 \%$ contraction intensity $(p<0.001)$ but was similar between young and older adults. Despite a significant interaction $(p=0.027)$, post-hoc tests did not reveal a different influence of contraction intensity between young and older adults (Fig. 3A-C). Except for four participants, a clear overestimation of the force to reproduce was evident for the $5 \%$ but not for the $20 \%$ 
MVC contraction intensity. Neither AE nor SE was associated with the raw force (in newtons) produced during the task for the $5 \%$ and the $20 \%$ contraction intensity $\left(r^{2}<0.10\right)$.

The aEMG for the FCR was similar between young and older adults for the 5\% [young: 7.0 (3.4)\% MVC, older: $8.5(3.1) \%$ MVC; $p=0.18]$ and the $20 \%$ contraction intensity [young: 20.5 (8.3)\% MVC, older: 20.4 (9.1)\% MVC; $p=0.96]$. In contrast, the aEMG for the ECR was greater in older adults [4.5 (2.2)\% MVC] than in young adults [2.9 $(1.2 \% \mathrm{MVC}]$ for the $5 \%$ contraction intensity $(p=0.012)$ but similar between groups for the $20 \%$ contraction intensity [young: 5.4 (3.9)\% MVC, older: 6.5 (3.1)\% MVC; $p=0.97$ ]. The coactivation index was similar between young and older adults for the 5\% [young: 24.4 (17.7)\%, older: 25.5 (9.7)\%; $p=0.83$ ] and $20 \%$ contraction intensities [young: 23.1 (7.7), older: $24.9(7.4) ; p=0.48]$.

When expressed as a percentage of its value during the target phase, the aEMG for the FCR, ECR and the coactivation index recorded during the reproduction phase were greater for the $5 \%$ than the $20 \%$ contraction intensity ( $p$ values $\leq 0.014$; Table 1 ), but did not differ between young and older adults $(p \geq 0.020)$.

\section{Effect of $L V$ on force reproduction task}

When vibration was applied during the reproduction phase, $\mathrm{AE}_{\mathrm{LV}}$ was greater for the 5\% [43.1(32.0)\%] than the 20\% contraction intensity [18.6 (14.7)\%; $(p<0.001)]$ and was greater for older [41.7 (32.4)\%] than for young adults [20.0 (16.1)\%; $(p<0.001)$; Fig. 3B-D]. Similarly, $\mathrm{SE}_{\mathrm{LV}}$ was greater for the $5 \%$ [34.9 (40.3)\%] than the $20 \%$ contraction intensity [7.8 (21.5)\%; Fig. 3B-D; $(p<0.001)]$ and greater for older [36.4 (37.7)\%] compared for young adults [6.3 (23.9)\%; $(p<0.001)]$.

\section{Discussion}

The results indicate that the force reproduction error was greater for the 5\% than the $20 \%$ contraction intensity regardless of age and was worsened when LV was applied. Furthermore, the reproduction error was greater in older than young adults for the $5 \%$ but not the $20 \%$ contraction intensity. The age difference in force reproduction error was exacerbated with LV.

\section{The magnitude of the force reproduction error varies with the contraction intensity}

As observed previously for a pinch grip task (De Serres and Fang 2004; Li et al. 2020), the magnitude of the force reproduction error was greater for the 5\% than the $20 \%$ contraction intensity. The greater error at 5\% MVC is characterised by an overestimation of the force to reproduce in most of the participants (90\% of the sample). A similar observation was reported for force-matching task when an external load was applied to index finger (Shergill et al. 2003; Voss et al. 2007; Walsh et al. 2011). However, this overestimation was abolished when both the target and the matching force were self-generated (Shergill et al. 2003; Walsh et al. 2011), as it was the case in the force reproduction task used in the present study. This supports the rationale that force matching task and force reproduction task do not involve similar mechanisms. Accordingly, it is difficult to properly discuss the present results based on force matching task experiments.

Previous work already noticed such an overestimation for low contraction levels during force reproduction tasks (De Serres and Fang 2004; Onneweer et al. 2016; Li et al. 2020), which may reflect the absence of visual information in the reproduction phase. This requires the central nervous system to increase the weight of proprioceptive and somaesthetic sources, which are noisy and have limited accuracy (Kording and Wolpert 2006). During low-force contraction, mechanotransducers, especially Golgi tendon organs, should be less loaded compared with higher force levels. It can, therefore, be expected that low sensory signals from Golgi tendon organs do not allow an accurate estimate of the force, especially when considering that the sensory signals are corrupted by neuronal noise (Bays and Wolpert 2007). To improve the proprioceptive acuity, however, the central nervous system could load a greater number of Golgi tendon organs by increasing the force produced by the muscle, leading to produce more force than required. Another possibility, if muscle spindles contribute to the sense of force (Monjo et al. 2018), would be to increase the fusimotor activity through the alphagamma coactivation (Allen et al. 2008). As the recruitment of gamma motor neurones is not proportional to that of alpha motor neurones (Wilson et al. 1997; Kakuda and Nagaoka 1998; Allen et al. 2008), a slight increase in motor command could result in a large change in fusimotor activity and thereby in proprioceptive inputs. Although such a strategy is not optimal as it induces an error in the force to reproduce, it may compensate, in part, for the absence of vision. In line with this hypothesis, Allen et al. (2008) reported that most of the fusimotor fibres are activated for contraction intensities lesser than $10 \%$ of the maximal force in the elbow flexors. This should induce a better force perception at 20\% than at 5\% MVC force, reducing the need to increase the fusimotor activity during the reproduction phase at $20 \%$ MVC. Finally, the mechanical pressure on the skin should be greater for the $20 \%$ than the 5\% contraction intensity, increasing thereby the signal-to-noise ratio of the somaesthetic modality at the higher force level. Further work is, however, needed to 
unravel the mechanisms underlying poorer force reproduction performance for low-force contractions.

\section{Local vibration increases the force reproduction error}

When applied to a muscle or tendon during a contraction, mechanical vibration of sufficient amplitude $(0.5-1 \mathrm{~mm})$ and frequency $(>20 \mathrm{~Hz}$ ) activates muscle spindles and Golgi tendon organs (Roll et al. 1989; Fallon and Macefield 2007), altering thereby the proprioceptive acuity (Goodwin et al. 1972; Cordo et al. 1995). The relevance of using LV to investigate the role of proprioception in the force reproduction performance was highlighted by Boucher et al. (Boucher et al. 2015), who showed that LV applied to the Achilles tendon altered the accuracy of force reproduction performed with the plantar flexor muscles in young adults. However, the nature of the task performed (ballistic contraction), does not allow to draw a finite conclusion on the effect of proprioceptive perturbation on the force reproduction performance. In our experimental conditions, LV exacerbated the force reproduction error (overestimation) at 5\% MVC, which may in part reflect an increase in muscle activation resulting from Ia and II afferents activation (Roll et al. 1989). However, Spiliopoulou and colleagues (2012) showed that LV applied during voluntary contractions did not significantly increase the force produced for contraction intensity below 20\% MVC. Nonetheless, the LV-induced increase in muscle afferents from mechanoreceptors (Roll et al. 1989; Fallon and Macefield 2007) may have enhanced the difficulty to process the proprioceptive information, as LV likely overwhelms the genuine proprioceptive inputs arising from the contraction. Such an effect should be more pronounced for low-force contractions during which mechanoreceptors, especially Golgi tendon organs, should be less loaded compared with higher contraction forces (see previous section). Regardless of the exact mechanisms, the results support the contribution of proprioceptive inputs from muscle spindles and tendon organs in the force reproduction performance.

\section{Ageing decreases the force reproduction performance during low-force contraction}

Two previous studies investigated the effect of ageing on force reproduction performance, with one study reporting a lesser performance for low-force contraction during pinch grip task in older adults (De Serres and Fang 2004), whereas the other did not find any age-related difference for ankle plantar flexors (Franco et al. 2015). As De Serres and Fang (2004), we observed an age effect for AE at low (5\% MVC) but not moderate contraction intensity (20\%
MVC). These results indicate that, in wrist flexors, the effect of healthy ageing on force reproduction performance is limited to low contraction force. As the older adults were weaker than young adults, the raw target force (in Newtons) was lesser in older adults, which could have made the task more difficult due to the very low force to produce. However, the reproduction error was not associated $\left(r^{2}<0.10\right)$ with the raw target force. Conversely, the increase in force reproduction error with ageing may reflect a decline in proprioceptive acuity (Goble et al. 2009; Henry and Baudry 2019), resulting from structural alterations (Shaffer and Harrison 2007) and decline in cortical processing of the proprioceptive signal (Piitulainen et al. 2018). In addition, older adults exhibited an increase in the two-point discrimination threshold (less tactile sensitivity), as observed previously (Bowden and McNulty 2013). Consequently, this sensory modality should provide less accurate information, particularly for the 5\% contraction intensity. It is to note, however, an apparent greater inter-individual variability in the older compared with the young group (Fig. 3), which indicates that the age-related decrease in the force reproduction performance may largely vary across the ageing population.

The results on the effect of ageing on the force reproduction performance could be confounded by age-related changes in short-term motor memory (Toole et al. 1984). However, Jordan (1978) reported no age-related difference in reproducing a movement with the arm after a rest period briefer than $50 \mathrm{~s}$. Furthermore, an influence of short-term motor memory cannot really explained the age effect only observed for the low contraction intensity. If the present results cannot completely rule out an effect of a decline in motor memory on the present results, such an effect could be assumed as marginal.

It has been suggested that the age-related decline in proprioception could be partly compensated by an increase in antagonist coactivation (Baudry et al. 2010; Nagai et al. 2011). Even if the coactivation index did not differ during the target phase between young and older adults, the aEMG of ECR was greater in older than young adults (4.5 vs. $2.9 \% \mathrm{MVC}$ ) for the $5 \%$ contraction intensity only. This may reflect an age-related trend to fall back on greater antagonist activation to control low force levels, as previously observed for forearm muscles (Baudry et al. 2010; Arellano et al. 2016). However, the level of aEMG of ECR during the reproduction phase, when expressed relative to its value recorded during the TARGET phase, did not differ between young and older adults. Such a result indicates that the reproduction phase did not require older adults to increase the level of antagonist coactivation. 


\section{Local vibration exacerbates force reproduction error in older adults}

Our results indicate a greater LV effect on force reproduction error in older adults. These results should not rely on an increased sensitivity of the muscle spindle pathway to LV in older adults as previous work reported a reduction in LV effect on tendon reflex and Jendrassik manoeuvre in older compared with young adults (Burke et al. 1996). Furthermore, Mildren et al. (2020) recently showed a lesser soleus EMG activity in response to LV in older compared with young adults, which was interpreted as a reduction in muscle spindle sensitivity to LV. It should be mentioned that those results were obtained in plantar flexor muscles and could, therefore, differ from upper limb muscles, although no evidence supports such a limb-related difference. Accordingly, the greater LV effect on the force reproduction performance in older adults likely reflects an age-related alteration in the processing of the proprioceptive signal (Goble et al. 2009, 2011; Mildren et al. 2020), which should impair the ability to discriminate and process the genuine proprioceptive signal originating from the muscle contraction from those arising from LV. This could be worsened by a possible age-related decrease in the signal-to-noise ratio within the central nervous system (Boisgontier and Swinnen 2015). The alterations observed in Golgi tendon organs should not induce a greater sensitivity of these mechanoreceptors to LV (Shaffer and Harrison 2007), but it may contribute to increase the difficulty to process the signals from these sensors, although no scientific evidence exists on such an effect.

\section{Methodological considerations}

Methodological aspects of this study should be discussed. First, tactile sensitivity was assessed at the fingertip level and not the palm used by the participants to exert force. Even though the fingertips have a greater tactile spatial resolution than the palm (Craig and Lyle 2001; Bowden and McNulty 2013), performance of spatial tasks discrimination (letter identification) on the palm can be predicted quantitatively from fingertip data. Furthermore, Bowden and McNulty (Bowden and McNulty 2013) reported that the age-related decrease in two-point discrimination sensitivity was similar between the palm and the fingertip. Therefore, the assessment of cutaneous sensitivity at the fingertip may not have drastically influenced our results on the tactile acuity.

Second, the moderate difference between young and older adults in force reproduction performance may arise from the fact that the older adults recruited in the present study were healthy and physically active. Furthermore, the range of age [from 60 to 90 years, 71 (7) years] and the mean age [71 (7) year] of the older group indicate that they do not belong to the oldest older. If this recruitment bias allows to consider the effect of healthy ageing on force reproduction task, further work is needed to investigate the sense of force in the oldest older.

\section{Conclusion}

This study provides elements supporting the rationale that proprioception contributes to the sense of force, as highlighted by the effect of tendon vibration on a force reproduction task. Furthermore, the results show a decrease in the force reproduction performance with ageing, which seems to be restricted to low force levels. The age effect on the force reproduction performance could reflect a decline in sensorimotor integration of proprioceptive and somaesthetic inputs, which alters the sense of force.

Supplementary Information The online version contains supplementary material available at https://doi.org/10.1007/s00221-022-06311-z.

Acknowledgements Mélanie Henry is currently supported by a grant of the "Fonds National de la Recherche Scientifique - FRS-FNRS", (FNRS, 2017-J1812640-206591) of Belgium.

Author contributions All authors contributed to the study conception and design. Material preparation, data collection and analysis were performed by $\mathrm{MH}$. The first draft of the manuscript was written by $\mathrm{MH}$ and all authors commented on previous versions of the manuscript. All authors read and approved the final manuscript.

Data availability The datasets generated during and/or analysed during the current study are available from the corresponding author on reasonable request.

\section{Declarations}

Conflict of interest The authors declare no competing financial interests.

\section{References}

Allen TJ, Ansems GE, Proske U (2008) Evidence from proprioception of fusimotor coactivation during voluntary contractions in humans. Exp Physiol 93:391-398. https://doi.org/10.1113/expph ysiol.2007.040741

Arellano CJ, Caha D, Hennessey JE, Amiridis IG, Baudry S, Enoka RM (2016) Fatigue-induced adjustment in antagonist coactivation by old adults during a steadiness task. J Appl Physiol 120:1039_ 1046. https://doi.org/10.1152/japplphysiol.00908.2015

Baudry S, Maerz AH, Enoka RM (2010) Presynaptic modulation of Ia afferents in young and old adults when performing force and position control. J Neurophysiol 103:623-631. https://doi.org/10. 1152/jn.00839.2009

Bays PM, Wolpert DM (2007) Computational principles of sensorimotor control that minimize uncertainty and variability. J Physiol 578:387-396. https://doi.org/10.1113/jphysiol.2006.120121 
Boisgontier MP, Swinnen SP (2015) Age-related deficit in a bimanual joint position matching task is amplitude dependent. Front Aging Neurosci 7:162. https://doi.org/10.3389/fnagi.2015.00162

Boucher JA, Normand MC, Boisseau E, Descarreaux M (2015) Sensorimotor control during peripheral muscle vibration: an experimental study. J Manip Physiol Ther 38:35-43. https://doi.org/10. 1016/j.jmpt.2014.10.013

Bowden JL, McNulty PA (2013) Age-related changes in cutaneous sensation in the healthy human hand. Age (dordr) 35:1077-1089. https://doi.org/10.1007/s11357-012-9429-3

Burke D, Hagbarth KE, Lofstedt L, Wallin BG (1976) The responses of human muscle spindle endings to vibration during isometric contraction. J Physiol 261:695-711. https://doi.org/10.1113/jphys iol.1976.sp011581

Burke JR, Schutten MC, Koceja DM, Kamen G (1996) Age-dependent effects of muscle vibration and the Jendrassik maneuver on the patellar tendon reflex response. Arch Phys Med Rehabil 77:600 604. https://doi.org/10.1016/s0003-9993(96)90302-0

Cordo P, Gurfinkel VS, Bevan L, Kerr GK (1995) Proprioceptive consequences of tendon vibration during movement. J Neurophysiol 74:1675-1688. https://doi.org/10.1152/jn.1995.74.4.1675

Craig JC, Lyle KB (2001) A comparison of tactile spatial sensitivity on the palm and fingerpad. Percept Psychophys 63:337-347. https:// doi.org/10.3758/bf03194474

De Serres SJ, Fang NZ (2004) The accuracy of perception of a pinch grip force in older adults. Can J Physiol Pharmacol 82:693-701. https://doi.org/10.1139/y04-085

Enoka RM, Christou EA, Hunter SK, Kornatz KW, Semmler JG, Taylor AM, Tracy BL (2003) Mechanisms that contribute to differences in motor performance between young and old adults. J Electromyogr Kinesiol 13:1-12. https://doi.org/10.1016/s10506411(02)00084-6

Fallon JB, Macefield VG (2007) Vibration sensitivity of human muscle spindles and Golgi tendon organs. Muscle Nerve 36:21-29. https://doi.org/10.1002/mus.20796

Fox EJ, Baweja HS, Kim C, Kennedy DM, Vaillancourt DE, Christou EA (2013) Modulation of force below $1 \mathrm{~Hz}$ : age-associated differences and the effect of magnified visual feedback. PLoS ONE 8:e55970. https://doi.org/10.1371/journal.pone.0055970

Franco PG, Santos KB, Rodacki AL (2015) Joint positioning sense, perceived force level and two-point discrimination tests of young and active elderly adults. Braz J Phys Ther 19:304-310. https:// doi.org/10.1590/bjpt-rbf.2014.0099

Goble DJ, Coxon JP, Wenderoth N, Van Impe A, Swinnen SP (2009) Proprioceptive sensibility in the elderly: degeneration, functional consequences and plastic-adaptive processes. Neurosci Biobehav Rev 33:271-278. https://doi.org/10.1016/j.neubiorev.2008.08.012

Goble DJ, Coxon JP, Van Impe A, Geurts M, Doumas M, Wenderoth N, Swinnen SP (2011) Brain activity during ankle proprioceptive stimulation predicts balance performance in young and older adults. J Neurosci 31:16344-16352. https://doi.org/10.1523/ JNEUROSCI.4159-11.2011

Goodwin GM, McCloskey DI, Matthews PB (1972) The contribution of muscle afferents to kinaesthesia shown by vibration induced illusions of movement and by the effects of paralysing joint afferents. Brain 95:705-748. https://doi.org/10.1093/brain/95.4.705

Henry M, Baudry S (2019) Age-related changes in leg proprioception: implications for postural control. J Neurophysiol 122:525-538. https://doi.org/10.1152/jn.00067.2019

Hunter SK, Pereira HM, Keenan KG (2016) The aging neuromuscular system and motor performance. J Appl Physiol 121:982-995. https://doi.org/10.1152/japplphysiol.00475.2016

Jordan T (1978) Age differences in visual and kinesthetic short-term memory. Percept Mot Skills 46:667-674. https://doi.org/10.2466/ pms.1978.46.2.667
Kakuda N, Nagaoka M (1998) Dynamic response of human muscle spindle afferents to stretch during voluntary contraction. J Physiol 513(Pt 2):621-628. https://doi.org/10.1111/j.1469-7793.1998. $621 \mathrm{bb} . \mathrm{x}$

Klass M, Baudry S, Duchateau J (2005) Aging does not affect voluntary activation of the ankle dorsiflexors during isometric, concentric, and eccentric contractions. J Appl Physiol 99:31-38. https:// doi.org/10.1152/japplphysiol.01426.2004

Klass M, Baudry S, Duchateau J (2008) Age-related decline in rate of torque development is accompanied by lower maximal motor unit discharge frequency during fast contractions. J Appl Physiol 104:739-746. https://doi.org/10.1152/japplphysiol.00550.2007

Kording KP, Wolpert DM (2006) Bayesian decision theory in sensorimotor control. Trends Cogn Sci 10:319-326. https://doi.org/10. 1016/j.tics.2006.05.003

Li L, Li Y, Wang H, Chen W, Liu X (2020) Effect of force level and gender on pinch force perception in healthy adults. Iperception 11:2041669520927043. https://doi.org/10.1177/2041669520 927043

Luft AR, Smith GV, Forrester L et al (2002) Comparing brain activation associated with isolated upper and lower limb movement across corresponding joints. Hum Brain Mapp 17:131-140. https://doi.org/10.1002/hbm.10058

Mildren RL, Schmidt ME, Eschelmuller G, Carpenter MG, Blouin JS, Inglis JT (2020) Influence of age on the frequency characteristics of the soleus muscle response to Achilles tendon vibration during standing. J Physiol 598:5231-5243. https://doi.org/10.1113/ JP280324

Monjo F, Shemmell J, Forestier N (2018) The sensory origin of the sense of effort is context-dependent. Exp Brain Res 236:19972008. https://doi.org/10.1007/s00221-018-5280-9

Nagai K, Yamada M, Uemura K, Yamada Y, Ichihashi N, Tsuboyama T (2011) Differences in muscle coactivation during postural control between healthy older and young adults. Arch Gerontol Geriatr 53:338-343. https://doi.org/10.1016/j.archger.2011.01.003

Oldfield RC (1971) The assessment and analysis of handedness: the Edinburgh inventory. Neuropsychologia 9:97-113. https://doi.org/ 10.1016/0028-3932(71)90067-4

Onneweer B, Mugge W, Schouten AC (2016) Force reproduction error depends on force level, whereas the position reproduction error does not. IEEE Trans Haptics 9:54-61. https://doi.org/10.1109/ TOH.2015.2508799

Piitulainen H, Seipajarvi S, Avela J, Parviainen T, Walker S (2018) Cortical proprioceptive processing is altered by aging. Front Aging Neurosci 10:147. https://doi.org/10.3389/fnagi.2018.00147

Proske U, Allen T (2019) The neural basis of the senses of effort, force and heaviness. Exp Brain Res 237:589-599. https://doi.org/10. 1007/s00221-018-5460-7

Proske U, Gandevia SC (2012) The proprioceptive senses: their roles in signaling body shape, body position and movement, and muscle force. Physiol Rev 92:1651-1697. https://doi.org/10.1152/physr ev.00048.2011

Roll JP, Vedel JP, Ribot E (1989) Alteration of proprioceptive messages induced by tendon vibration in man: a microneurographic study. Exp Brain Res 76:213-222. https://doi.org/10.1007/BF00253639

Shaffer SW, Harrison AL (2007) Aging of the somatosensory system: a translational perspective. Phys Ther 87:193-207. https://doi.org/ 10.2522/ptj.20060083

Shergill SS, Bays PM, Frith CD, Wolpert DM (2003) Two eyes for an eye: the neuroscience of force escalation. Science 301:187. https:// doi.org/10.1126/science.1085327

Spiliopoulou SI, Amiridis IG, Hatzitaki V, Patikas D, Kellis E (2012) Tendon vibration during submaximal isometric strength and postural tasks. Eur J Appl Physiol 112:3807-3817. https://doi.org/10. 1007/s00421-012-2319-7 
Toole T, Pyne A, McTarsney PA (1984) Age differences in memory for movement. Exp Aging Res 10:205-210. https://doi.org/10.1080/ 03610738408258466

Voss M, Bays PM, Rothwell JC, Wolpert DM (2007) An improvement in perception of self-generated tactile stimuli following theta-burst stimulation of primary motor cortex. Neuropsychologia 45:2712 2717. https://doi.org/10.1016/j.neuropsychologia.2007.04.008

Walsh LD, Taylor JL, Gandevia SC (2011) Overestimation of force during matching of externally generated forces. J Physiol 589:547557. https://doi.org/10.1113/jphysiol.2010.198689

Wilson LR, Gandevia SC, Burke D (1997) Discharge of human muscle spindle afferents innervating ankle dorsiflexors during target isometric contractions. J Physiol 504(Pt 1):221-232. https://doi. org/10.1111/j.1469-7793.1997.221bf.x

Woolcott JC, Richardson KJ, Wiens MO, Patel B, Marin J, Khan KM, Marra CA (2009) Meta-analysis of the impact of 9 medication classes on falls in elderly persons. Arch Intern Med 169:19521960. https://doi.org/10.1001/archinternmed.2009.357

Publisher's Note Springer Nature remains neutral with regard to jurisdictional claims in published maps and institutional affiliations. 